\title{
A Research Travelogue Towards Educational Data Mining
}

\author{
Bernard Ugalde \\ AMA University Online Education \\ Quezon City, Philippines
}

\author{
R. Venkateswaran \\ Salalah College of Technology \\ Salalah, OMAN
}

\begin{abstract}
In the recent era of technology, educational institutions innovate itself to find new ways to serve its educational community efficiently and effectively. Information systems have been there for quite some time as the backbone of education institutions to support its daily operations. At this point, educational databases have much information but remain utilized. In order to make benefit from such big data, a power tool is required like data mining for analysis and prediction. Data mining has been proven useful in various aspects of our lives like in advertising, marketing, loans and now a new frontier in the field of education. It has been noted that there is no unified approach among researchers in educational data mining and a considerable amount of work is required towards this field. This research presents a comprehensive travelogue (2010- 2017) in educational data mining with respect to related international journals available from various sources, and secondary data collected from the organization in the form of survey reports.
\end{abstract}

\section{Keywords}

Educational Data Mining, Trends in EMD, Future vision of EDM

\section{INTRODUCTION}

In the last decade, educational institutions have been continuously innovating itself to cope up with the fastchanging industry needs. They employ qualified staff, create state-of-the-art laboratories for practice and simulation, develop and employ a tool that will help in the administration and management of schools. However, with all of these measures in place, schools are still confronted with problems of student's dropout, poor academic performance, and unemployment of graduates.

Although educational institutions collect and keep student records, it has been noted that most of the time these data and information are not utilized properly. Data are just collected every academic year, and are kept in filing cabinets in school vaults, school's information systems or dump somewhere.

Analyzing and understanding factors that affect the number of dropouts, monitoring academic performance, keeping track of alumni employment in education institution entails complex, tedious and endless. Academic marks are maintained by these organizations for purpose of printing student's transcript later. Data on a number of enrollments and analysis on student's demographics and background are unutilized and does not support any decision or policymaking within institutions. Thus, in order for these problems to be addressed, a very useful tool for an educational institution is needed. There must be a way to analyze, understand and predict performance needs of students scientifically, and a way to inform and support the whole educational community.
Only if universities could get a clear picture of the current standing of the entire student populations of the institution that will help them make pro-active measures to form of policies and programs that will align and address the needs of the students and the whole school community. This will promote efficient and effective learning on the side of the student. On the teacher's side, they can plan ahead with their delivery inside the classroom. School management will get its benefit by attracting a great number of enrollees. As a whole getting the quality education that each deserved. In the first place, the genuine goal of each educational institution is to let the student learn and let them add value to the workforce later.

Educational data mining have shown noteworthy advantages to education institutions It is a very powerful tool to reveal the pattern and precious knowledge, which otherwise may not be identified. It also helps to respond to educational question and problems that might be needed data analysis and interpretation. Substantial work has been done towards the usage of data mining techniques in education, but still, there are many untouched areas and no unified approach is followed.

This paper presents a comprehensive literature review of relevant researches done in educational data mining from 2010 to 2017. Section 2 gives a short note about educational data mining. Section 3 presents the review of related researchers in a form of a table. Section 4, 5 and 6 presents the research objectives, methodology, and findings of this study respectively, followed by conclusions and recommendation, and references.

\section{A BRIEF NOTE ABOUT EDUCATIONAL DATA MINING}

The following discusses educational data mining in several areas as follows:

\subsection{What is Educational Data Mining?}

According to International Educational Data Mining Society [1], Educational Data Mining (EDM) is an emerging discipline, concerned with developing methods for exploring the unique and increasingly large-scale data that come from educational settings and using those methods to better understand students, and the settings which they learn in. EDM focuses on developing new tools and algorithm for discovering data patterns [2]. Particularly in education settings, it can answer questions like (1) what sequences of topics is most effective for a specific student? (2) Which student action is associated with better learning and higher grades? (3) Which action indicates satisfaction and engagement? (4) What features of an online learning environment lead to better learning?

\subsection{Goals of Educational Data Mining}

Table 1 below presents the goal of educational data mining. EDM can predict student future learning behavior and can 
determine the optimal instructional sequences to support the student's learning style. It provides a clear picture of the academic performance of a student and determines the needed support. Finally, EDM test learning theories and inform its stakeholders about the effectiveness and efficiency of the whole teaching-learning process. All of these relates to the direct participation of the student in teaching and learning process which falls under EDM's student-oriented goal.

Table 1 Goals of EDM

\begin{tabular}{|c|l|}
\hline SN & \multicolumn{1}{|c|}{ Goal } \\
\hline 1 & Predict student's future learning behavior. \\
\hline 2 & $\begin{array}{l}\text { Determine the optimal instructional sequences to } \\
\text { support the student's learning style. }\end{array}$ \\
\hline 3 & $\begin{array}{l}\text { Provide a clear picture of the academic performance } \\
\text { of a student. }\end{array}$ \\
\hline 4 & $\begin{array}{l}\text { Determine the needed academic support of the } \\
\text { student, and assess if the support given is effective }\end{array}$ \\
\hline 5 & $\begin{array}{l}\text { Provide feedback and adapt learning } \\
\text { recommendations for behavior }\end{array}$ \\
\hline 6 & $\begin{array}{l}\text { Test and evaluate the learning theories and learning } \\
\text { materials. }\end{array}$ \\
\hline 7 & Evaluates the whole teaching-learning process \\
\hline
\end{tabular}

\section{A. Educational Data Mining Process}

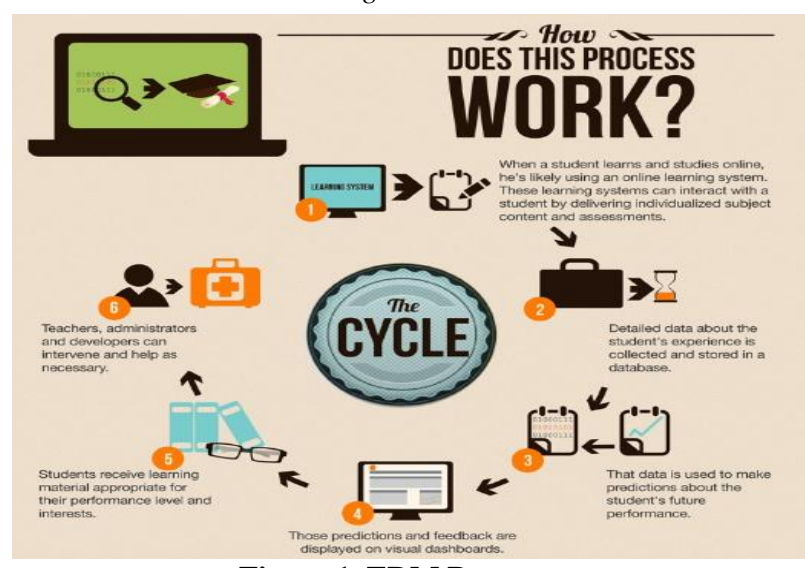

Figure 1. EDM Process

According to [3], EDM is viewed as a cycle. It starts with data collection and pre-processing. Students are directed to use the online learning system. Delivery of the course is based on individualized subject content and assessment metrics. Detailed student's performance and experience are recorded and stored in the database. The student information is transformed into models that are used to predict the future learning behavior and feedback using dashboard. A student will receive learning materials based on their performance level and learning style. Along the ways, other stakeholders can intervene to mitigate any issues or difficulties encountered. The whole EDM process is illustrated in figure 1.

\subsection{Educational Data Mining Stakeholders}

There are generally three stakeholders in EDM but can change based on the size of the organization. Teachers prepare and develop the course materials and set the assessment metric. They mentor the students by monitoring student's academic performance and assessing their needs. They facilitate the learning process of the student. In a smaller organization, teachers are also tasked to prepare the course objectives and goals. They will also analyze the whole student's experience in the database and provide necessary actions. Students will undergo the learning process by accessing the learning materials and assessments. At the end, he then gives feedback and recommendation to the learning process as a whole. Administrators and management set priorities, approve plans, support the cost incurred in from the conceptualization of the EDM to its implementation and maintenance. Table 2 summarizes the stakeholders of EDM.

\begin{tabular}{|c|l|}
\multicolumn{2}{|c}{ Table 2. Stakeholders of EDM } \\
\hline Stakeholder & \multicolumn{1}{|c|}{ Role } \\
\hline \multirow{5}{*}{ Teachers } & $\begin{array}{l}\text { Prepares and develop the course materials } \\
\text { and sets the assessment metric. } \\
\text { Mentors the students by monitoring } \\
\text { student's academic performance and } \\
\text { assessing their needs. } \\
\text { Facilitate the learning process of the } \\
\text { student. } \\
\text { Prepare the course objectives and goals. } \\
\text { Analyze the whole student's experience in } \\
\text { the database and provide necessary } \\
\text { actions. }\end{array}$ \\
\hline Students & $\begin{array}{l}\text { Undergo the learning process by accessing } \\
\text { the learning materials and assessments. }\end{array}$ \\
$\begin{array}{l}\text { Give feedback and recommendation to the } \\
\text { learning process as a whole. }\end{array}$ \\
\hline $\begin{array}{c}\text { Management } \\
\text { and }\end{array}$ & $\begin{array}{l}\text { Set priorities; approve plans support the } \\
\text { cost incurred in from the conceptualization } \\
\text { of the EDM to its implementation and } \\
\text { maintenance. }\end{array}$ \\
\hline
\end{tabular}

\subsection{EMD Methods and Techniques}

To discover knowledge from databases, various algorithms, methods, and techniques are used. The following DM methods are popular with the EDM research community.

1. Prediction is a technique which goal is to develop a model that can infer a single aspect of data from some combination of other aspects of data [4]. Generally, it has three types: classification, regression, and density estimation. Classification uses can use either categorical or binary input variables, while regression uses continuous input variables. Density estimation can be done with the help of various kernel functions.

2. Clustering divides data set into various groups, known as clusters [5]. It is best used if the data set is already specified. As per clustering phenomenon, the data point of one cluster and should be more similar to other data points of the same cluster and more dissimilar to data points of another cluster. There are two ways of initiation of a clustering algorithm. Firstly, start the clustering algorithm with no prior assumption and second is to start clustering algorithm with a prior postulate.

3. Relationship mining generally refers to contrive new relationships between variables, done on a large data set, having a no of variables [6]. It attempts to discover the variable which is most closely associated with the specified variable. There are four types of relationship mining: association rule mining, correlation mining, and sequential pattern mining and causal data mining. Association data mining is based on an if-then rule that is if some particular set of variable value appears then it generally has a specified value. In correlation mining, the linear correlations are discovered between variables. The aim of 
sequential pattern mining is to extract temporal relationships between variables.

4. Discovery with models includes the designing of model based on some of the basic concepts like prediction, clustering and knowledge engineering used to discover new predicted variables [7].

5. Distillation of data for human judgment by identifying and classifying the data in a way that human can easily recognize the pattern [8].

\subsection{EMD Tools}

The following table presents the popular tools use for educational data mining though there are many tools available right now on the market.

\begin{tabular}{|c|c|c|c|c|c|}
\hline Ref & $\begin{array}{c}\text { Name of } \\
\text { Tool and } \\
\text { Developer }\end{array}$ & $\begin{array}{c}\text { Licen } \\
\text { se }\end{array}$ & $\begin{array}{c}\text { Function } \\
\text { s and } \\
\text { Features } \\
\end{array}$ & $\begin{array}{c}\text { Techniq } \\
\text { ues and } \\
\text { Tools } \\
\end{array}$ & $\begin{array}{c}\text { Operat } \\
\text { ing } \\
\text { System }\end{array}$ \\
\hline [9] & $\begin{array}{l}\text { MSSQL } \\
\text { Server } \\
\text { (Microsoft) }\end{array}$ & $\begin{array}{l}\text { Com } \\
\text { merci } \\
\text { al }\end{array}$ & $\begin{array}{l}\text { Provides } \\
\text { DM } \\
\text { functions } \\
\text { both in } \\
\text { relational } \\
\text { db } \\
\text { system } \\
\text { and Data } \\
\text { Warehou } \\
\text { se } \\
\text { (DWH) } \\
\text { system } \\
\text { environm } \\
\text { ent. }\end{array}$ & $\begin{array}{l}\text { Integrates } \\
\text { the } \\
\text { algorithm } \\
\text { s } \\
\text { develope } \\
\text { d by third } \\
\text { party } \\
\text { vendors } \\
\text { and } \\
\text { applicatio } \\
\text { n users. }\end{array}$ & $\begin{array}{l}\text { Windo } \\
\text { ws, } \\
\text { Linux }\end{array}$ \\
\hline $\begin{array}{c}{[10} \\
]\end{array}$ & $\begin{array}{l}\text { Oracle } \\
\text { Data } \\
\text { Mining } \\
\text { (Oracle } \\
\text { Corp) }\end{array}$ & $\begin{array}{l}\text { Com } \\
\text { merci } \\
\text { al }\end{array}$ & $\begin{array}{l}\text { Provides } \\
\text { an } \\
\text { embedde } \\
\text { d DWH } \\
\text { infrastruc } \\
\text { ture for } \\
\text { multidim } \\
\text { ensional } \\
\text { data } \\
\text { analysis }\end{array}$ & $\begin{array}{l}\text { Associati } \\
\text { on } \\
\text { Mining, } \\
\text { Classifica } \\
\text { tion, } \\
\text { Predictio } \\
\text { n, } \\
\text { Regressio } \\
\text { n, } \\
\text { Clusterin } \\
\text { g, } \\
\text { Sequence } \\
\text { similarity } \\
\text { search } \\
\text { and } \\
\text { analysis. }\end{array}$ & $\begin{array}{l}\text { Windo } \\
\text { ws, } \\
\text { Mac, } \\
\text { Linux }\end{array}$ \\
\hline $\begin{array}{c}{[11} \\
]\end{array}$ & $\begin{array}{l}\text { SPSS } \\
\text { Clementine } \\
\text { (IBM) }\end{array}$ & $\begin{array}{l}\text { Com } \\
\text { merci } \\
\text { al }\end{array}$ & $\begin{array}{l}\text { Provides } \\
\text { an } \\
\text { integrate } \\
\text { d data } \\
\text { mining } \\
\text { develop } \\
\text { ment } \\
\text { environm } \\
\text { ent for } \\
\text { end users } \\
\text { and } \\
\text { develope } \\
\text { rs. }\end{array}$ & $\begin{array}{l}\text { Associati } \\
\text { on } \\
\text { Mining, } \\
\text { Clusterin } \\
\text { g, } \\
\text { Classifica } \\
\text { tion, } \\
\text { Predictio } \\
\text { n and } \\
\text { visualizat } \\
\text { ion tools }\end{array}$ & $\begin{array}{l}\text { Windo } \\
\text { ws, } \\
\text { Solaris, } \\
\text { Linux }\end{array}$ \\
\hline $\begin{array}{c}{[12} \\
]\end{array}$ & $\begin{array}{l}\text { WEKA } \\
\text { (University } \\
\text { of } \\
\text { Waikato, }\end{array}$ & $\begin{array}{l}\text { Open/ } \\
\text { free }\end{array}$ & $\begin{array}{l}\text { Provides } \\
\text { machine } \\
\text { learning } \\
\text { algorithm }\end{array}$ & $\begin{array}{l}\text { Data pre- } \\
\text { processin } \\
\mathrm{g}, \\
\text { classifica }\end{array}$ & $\begin{array}{l}\text { Windo } \\
\text { ws, } \\
\text { Linux }\end{array}$ \\
\hline
\end{tabular}

\begin{tabular}{|c|c|c|c|c|c|}
\hline & $\begin{array}{l}\text { New } \\
\text { Zealand) }\end{array}$ & & $\begin{array}{l}\text { s for data } \\
\text { mining } \\
\text { tasks. } \\
\text { Well- } \\
\text { suited for } \\
\text { developi } \\
\text { ng new } \\
\text { machine } \\
\text { learning } \\
\text { schemes. }\end{array}$ & $\begin{array}{l}\text { tion, } \\
\text { regressio } \\
\text { n, } \\
\text { clustering } \\
\text {, } \\
\text { associatio } \\
\text { n rules, } \\
\text { and } \\
\text { visualizat } \\
\text { ion. }\end{array}$ & \\
\hline $\begin{array}{c}{[13} \\
]\end{array}$ & $\begin{array}{l}\text { ORANGE } \\
\text { (University } \\
\text { of } \\
\text { Ljubljana, } \\
\text { Slovenia) }\end{array}$ & $\begin{array}{l}\text { Open/ } \\
\text { free }\end{array}$ & $\begin{array}{l}\text { Provides } \\
\text { open } \\
\text { source } \\
\text { data } \\
\text { visualizat } \\
\text { ion and } \\
\text { analysis } \\
\text { for } \\
\text { novice } \\
\text { and } \\
\text { experts }\end{array}$ & $\begin{array}{l}\text { Text } \\
\text { mining } \\
\text { and } \\
\text { Bioinfor } \\
\text { matics } \\
\text { add-on }\end{array}$ & $\begin{array}{l}\text { Windo } \\
\text { ws, } \\
\text { Linux }\end{array}$ \\
\hline $\begin{array}{c}{[14} \\
]\end{array}$ & Carrot & $\begin{array}{l}\text { Open/ } \\
\text { free }\end{array}$ & $\begin{array}{l}\text { Provides } \\
\text { ready-to- } \\
\text { use } \\
\text { compone } \\
\text { nts for } \\
\text { fetching } \\
\text { search } \\
\text { results } \\
\text { from } \\
\text { various } \\
\text { sources }\end{array}$ & $\begin{array}{l}\text { Clusterin } \\
\mathrm{g}\end{array}$ & $\begin{array}{l}\text { Windo } \\
\text { ws, } \\
\text { Linux }\end{array}$ \\
\hline
\end{tabular}

All these concepts and theories in EDM mentioned in this section will be used in the following section of the study.

\section{REVIEW OF RELATED LITERATURE}

A comprehensive literature survey of various significant researchers in the area of Educational Data Mining ranging from the year 2010 to 2017 is presented below (Table 4). The leverage points of this survey are trends of DM methods and respective key findings.

Table 4. Educational Data Mining Papers

\begin{tabular}{|c|l|l|l|}
\hline $\begin{array}{c}\text { Referenc } \\
\text { e }\end{array}$ & \multicolumn{1}{|c|}{$\begin{array}{c}\text { Year, } \\
\text { Author(s) }\end{array}$} & $\begin{array}{c}\text { Methodolog } \\
\text { y }\end{array}$ & Key Findings \\
\hline \multirow{5}{*}[15]{} & $\begin{array}{l}\text { 2017, Ms. } \\
\text { Ganesan } \\
\text { Kavitha, and } \\
\text { Dr. Lawrance } \\
\text { Raj }\end{array}$ & $\begin{array}{l}\text { Analyzed } \\
\text { students' } \\
\text { performance } \\
\text { in their } \\
\text { assessment to } \\
\text { discover the } \\
\text { students at } \\
\text { risk of failing } \\
\text { the final exam }\end{array}$ \\
\hline \multirow{5}{*}[16]{} & $\begin{array}{l}\text { Gain rato } \\
\text { ihák and } \\
\text { Radek } \\
\text { Pelánek }\end{array}$ & $\begin{array}{l}\text { Measured } \\
\text { techniques, } \\
\text { matrix } \\
\text { factorization } \\
\text { s and neural } \\
\text { educational of } \\
\text { items using } \\
\text { data on } \\
\text { learners' } \\
\text { performance }\end{array}$ \\
\hline \multirow{2}{*}[17]{} & $\begin{array}{l}\text { 2017, Aaron } \\
\text { Bauer, Jeff } \\
\text { Flatten and } \\
\text { Zoran }\end{array}$ & $\begin{array}{l}\text { Iterative } \\
\text { visualization } \\
\text {-based } \\
\text { methodolog }\end{array}$ & $\begin{array}{l}\text { Analyzed } \\
\text { problem- } \\
\text { solving } \\
\text { behavior in }\end{array}$ \\
\hline
\end{tabular}




\begin{tabular}{|c|c|c|c|}
\hline & Popovi'c & $\mathrm{y}$ & $\begin{array}{l}\text { open-ended } \\
\text { scientific- } \\
\text { discovery } \\
\text { game } \\
\text { challenges } \\
\end{array}$ \\
\hline$[18]$ & $\begin{array}{l}\text { 2017, Zhiyun } \\
\text { Ren, Xia } \\
\text { Ning, Huzefa } \\
\text { Rangwala }\end{array}$ & $\begin{array}{l}\text { factorization } \\
\text {-based } \\
\text { approach } \\
\text { called } \\
\text { Matrix } \\
\text { Factorizatio } \\
\text { n with } \\
\text { Temporal } \\
\text { Course-wise } \\
\text { Influence }\end{array}$ & $\begin{array}{l}\text { Predicted } \\
\text { Grade with } \\
\text { Temporal } \\
\text { Course-wise } \\
\text { Influence }\end{array}$ \\
\hline [19] & $\begin{array}{l}\text { 2017, Angela } \\
\text { Stewart, Nigel } \\
\text { Bosch and } \\
\text { Sidney K. } \\
\text { D'Mello }\end{array}$ & $\begin{array}{l}\text { Variety of } \\
\text { machine } \\
\text { learning } \\
\text { techniques }\end{array}$ & $\begin{array}{l}\text { Generalizes } \\
\text { Face-Based } \\
\text { Mind } \\
\text { Wandering } \\
\text { Detection } \\
\text { Across Task } \\
\text { Contexts } \\
\end{array}$ \\
\hline [20] & $\begin{array}{l}\text { 2017, Andrew } \\
\text { M. Olney, } \\
\text { Dariush } \\
\text { Bakhtiari, } \\
\text { Daphne } \\
\text { Greenberg } \\
\text { and Art } \\
\text { Graesser } \\
\end{array}$ & $\begin{array}{l}\text { Q-MATRIX } \\
\& \\
\text { LOGISTIC } \\
\text { MIXED } \\
\text { MODELS }\end{array}$ & $\begin{array}{l}\text { Assessed } \\
\text { computer } \\
\text { literacy of } \\
\text { adults with } \\
\text { low literacy } \\
\text { skills }\end{array}$ \\
\hline [21] & $\begin{array}{l}\text { 2017, Renu } \\
\text { Balyan, } \\
\text { Kathryn S. } \\
\text { McCarthy and } \\
\text { Danielle S. } \\
\text { McNamara } \\
\end{array}$ & $\begin{array}{l}\text { Random } \\
\text { Forests, } \\
\text { Trees and } \\
\text { Support } \\
\text { Vector } \\
\text { Machines } \\
\end{array}$ & $\begin{array}{l}\text { Assessed } \\
\text { Literary Text } \\
\text { Comprehensio } \\
\mathrm{n}\end{array}$ \\
\hline [22] & $\begin{array}{l}\text { 2016, Ankita } \\
\text { Katar and } \\
\text { Shubha } \\
\text { Dubey }\end{array}$ & $\begin{array}{l}\text { Classificatio } \\
\mathrm{n} \text { algorithms }\end{array}$ & $\begin{array}{l}\text { Evaluated } \\
\text { student } \\
\text { performance }\end{array}$ \\
\hline [23] & $\begin{array}{l}\text { 2016, Amjad } \\
\text { Abu Saa }\end{array}$ & $\begin{array}{l}\text { Multiple } \\
\text { classificatio } \\
\text { n methods }\end{array}$ & $\begin{array}{l}\text { Predicted the } \\
\text { students' } \\
\text { grade at the } \\
\text { end of the } \\
\text { semester }\end{array}$ \\
\hline [24] & $\begin{array}{l}2016, \\
\text { Alexandria K. } \\
\text { Vail, Joseph } \\
\text { B. Wiggins, } \\
\text { Joseph F. } \\
\text { Grafsgaard, } \\
\text { Kristy } \\
\text { Elizabeth } \\
\text { Boyer, Eric N. } \\
\text { Wiebe and } \\
\text { James C. } \\
\text { Lester }\end{array}$ & Regression & $\begin{array}{l}\text { Analyzed of } \\
\text { student } \\
\text { affective } \\
\text { response, as } \\
\text { evidenced } \\
\text { by multimodal } \\
\text { data streams, } \\
\text { immediately } \\
\text { following } \\
\text { tutor } \\
\text { questions. }\end{array}$ \\
\hline [25] & $\begin{array}{l}\text { 2016, Martin } \\
\text { Stapel, Zhilin } \\
\text { Zheng and } \\
\text { Niels } \\
\text { Pinkwart }\end{array}$ & $\begin{array}{l}\text { Specialized } \\
\text { scope } \\
\text { classifiers }\end{array}$ & $\begin{array}{l}\text { Predict } \\
\text { student } \\
\text { performance }\end{array}$ \\
\hline [26] & $\begin{array}{l}\text { 2016, Mirka } \\
\text { Saarela and } \\
\text { Tommi } \\
\text { K̈arkk äinen }\end{array}$ & $\begin{array}{l}\text { Robust } \\
\text { clustering } \\
\text { technique }\end{array}$ & $\begin{array}{l}\text { Analyzed } \\
\text { students' } \\
\text { overall } \\
\text { performance } \\
\end{array}$ \\
\hline
\end{tabular}

\begin{tabular}{|c|c|c|c|}
\hline & & & $\begin{array}{l}\text { based on } \\
\text { learning skill } \\
\text { and core } \\
\text { studies }\end{array}$ \\
\hline [27] & $\begin{array}{l}\text { 2016, Arjun } \\
\text { Sharma, Arijit } \\
\text { Biswas, Ankit } \\
\text { Gandhi, Sonal } \\
\text { Patil, Om } \\
\text { Deshmukh }\end{array}$ & $\begin{array}{l}\text { Neural } \\
\text { network }\end{array}$ & $\begin{array}{l}\text { predicted } \\
\text { liveliness in } \\
\text { educational } \\
\text { videos }\end{array}$ \\
\hline [28] & $\begin{array}{l}\text { 2016, Ke Niu, } \\
\text { Zhendong } \\
\text { Niu, Xiangyu } \\
\text { Zhao, Can } \\
\text { Wang, Kai } \\
\text { Kang, Min Ye }\end{array}$ & $\begin{array}{l}\text { Clustering } \\
\text { algorithms }\end{array}$ & $\begin{array}{l}\text { Analyzed } \\
\text { users' learning } \\
\text { behaviors and } \\
\text { help to } \\
\text { provide } \\
\text { personalized } \\
\text { learning } \\
\text { guides in } \\
\text { traditional } \\
\text { Web-based } \\
\text { learning } \\
\text { systems. }\end{array}$ \\
\hline [29] & $\begin{array}{l}\text { 2015, Engin } \\
\text { Bumbacher, } \\
\text { Shima Salehi, } \\
\text { Miriam } \\
\text { Wierzchula } \\
\text { and Paulo } \\
\text { Blikstein }\end{array}$ & $\begin{array}{l}\text { Cluster } \\
\text { analysis }\end{array}$ & $\begin{array}{l}\text { How learning } \\
\text { environment } \\
\text { and inquiry } \\
\text { behaviors } \\
\text { affects the } \\
\text { development } \\
\text { of conceptual } \\
\text { understanding } \\
\text { in Physics }\end{array}$ \\
\hline [30] & $\begin{array}{l}\text { 2015, Juraj } \\
\text { Nižnan, } \\
\text { Radek } \\
\text { Pelánek, Jir`í } \\
\text { R`ihák }\end{array}$ & $\begin{array}{l}\text { Bayesian } \\
\text { extension, a } \\
\text { hierarchical } \\
\text { model, and a } \\
\text { networked } \\
\text { model } \\
\end{array}$ & $\begin{array}{l}\text { Analyzed } \\
\text { Student } \\
\text { Models for } \\
\text { Prior } \\
\text { Knowledge } \\
\text { Estimation } \\
\end{array}$ \\
\hline [31] & $\begin{array}{l}\text { 2015, Yang } \\
\text { Chen, Pierre- } \\
\text { Henri } \\
\text { Wuillemin, } \\
\text { Jean-Marc } \\
\text { Labat }\end{array}$ & $\begin{array}{l}\text { Probabilistic } \\
\text { Association } \\
\text { Rules }\end{array}$ & $\begin{array}{l}\text { Discovering } \\
\text { Prerequisite } \\
\text { Structure of } \\
\text { Skills }\end{array}$ \\
\hline [32] & $\begin{array}{l}\text { 2015, Aysu } \\
\text { Ezen-Can and } \\
\text { Kristy } \\
\text { Elizabeth } \\
\text { Boyer }\end{array}$ & $\begin{array}{l}\text { Multiple } \\
\text { regression } \\
\text { models }\end{array}$ & $\begin{array}{l}\text { Identified the } \\
\text { factors that } \\
\text { may be } \\
\text { influential in } \\
\text { students' } \\
\text { levels of } \\
\text { interaction } \\
\text { with the } \\
\text { system }\end{array}$ \\
\hline [33] & $\begin{array}{l}\text { 2015, Mirka } \\
\text { Saarela, } \\
\text { Tommi } \\
\text { Kärkkäinen }\end{array}$ & $\begin{array}{l}\text { Clustering } \\
\text { Approach }\end{array}$ & $\begin{array}{l}\text { Determine if } \\
\text { country } \\
\text { stereotypes } \\
\text { exist in } \\
\text { Programme } \\
\text { for } \\
\text { International } \\
\text { Student } \\
\text { Assessment } \\
\text { (PISA) }\end{array}$ \\
\hline [34] & $\begin{array}{l}\text { 2014, Peña- } \\
\text { Ayala, } \\
\text { Alejandro }\end{array}$ & $\begin{array}{l}\text { Statistical } \\
\text { and } \\
\text { Clustering } \\
\text { Processes }\end{array}$ & $\begin{array}{l}\text { Identified } \\
\text { kinds of } \\
\text { educational } \\
\text { systems, }\end{array}$ \\
\hline
\end{tabular}




\begin{tabular}{|c|c|c|c|}
\hline & & & $\begin{array}{l}\text { disciplines, } \\
\text { tasks, } \\
\text { methods, and } \\
\text { algorithms. }\end{array}$ \\
\hline [35] & $\begin{array}{l}2014, \\
\text { Saranya, S., } \\
\text { R. Ayyappan, } \\
\text { and N. Kumar }\end{array}$ & $\begin{array}{l}\text { Naive Bayes } \\
\text { Algorithm }\end{array}$ & $\begin{array}{l}\text { Graphically } \\
\text { represented } \\
\text { institutional } \\
\text { growth } \\
\text { prognosis and } \\
\text { students' } \\
\text { progress } \\
\text { analysis. }\end{array}$ \\
\hline [36] & $\begin{array}{l}\text { 2014, Archer, } \\
\text { Elizabeth, } \\
\text { Yuraisha } \\
\text { Bianca } \\
\text { Chetty, and } \\
\text { Paul Prinsloo }\end{array}$ & $\begin{array}{l}\text { Experimenta } \\
1 \text { Usage of } \\
\text { Employee } \\
\text { Profiling } \\
\text { Software }\end{array}$ & $\begin{array}{l}\text { Experimented } \\
\text { the usage of a } \\
\text { commercial } \\
\text { product } \\
\text { generally used } \\
\text { for employee } \\
\text { profiling in } \\
\text { corporate, for } \\
\text { higher } \\
\text { education } \\
\text { environment. }\end{array}$ \\
\hline [37] & $\begin{array}{l}\text { 2014, Hicheur } \\
\text { Cairns, } \\
\text { Awatef, et al. }\end{array}$ & $\begin{array}{l}\text { Clustering } \\
\text { Technique }\end{array}$ & $\begin{array}{l}\text { Professionals' } \\
\text { data was } \\
\text { analyzed } \\
\text { during } \\
\text { training of a } \\
\text { consulting } \\
\text { company. }\end{array}$ \\
\hline [38] & $\begin{array}{l}\text { 2014, Arora, } \\
\text { Rakesh and } \\
\text { Dharmendra } \\
\text { Badal }\end{array}$ & $\begin{array}{l}\text { Association } \\
\text { Analysis } \\
\text { Algorithm }\end{array}$ & $\begin{array}{l}\text { Found set of } \\
\text { weak students } \\
\text { based on } \\
\text { graduation } \\
\text { and post- } \\
\text { graduation } \\
\text { marks. }\end{array}$ \\
\hline [39] & $\begin{array}{l}\text { 2013, Stephen } \\
\text { E. Fancsali, } \\
\text { Tristan Nixon, } \\
\text { and Steven } \\
\text { Ritter }\end{array}$ & $\begin{array}{l}\text { Bayesian } \\
\text { Knowledge } \\
\text { Tracing }\end{array}$ & $\begin{array}{l}\text { Optimal and } \\
\text { Worst-Case } \\
\text { Performance } \\
\text { of Mastery } \\
\text { Learning } \\
\text { Assessment } \\
\text { with Bayesian } \\
\text { Knowledge } \\
\text { Tracing }\end{array}$ \\
\hline [40] & $\begin{array}{l}\text { 2013, Joseph } \\
\text { F. Grafsgaard, } \\
\text { Joseph B. } \\
\text { Wiggins, } \\
\text { Kristy } \\
\text { Elizabeth } \\
\text { Boyer, Eric N. } \\
\text { Wiebe, James } \\
\text { C. Lester }\end{array}$ & $\begin{array}{l}\text { Bayesian } \\
\text { Information } \\
\text { Criterion }\end{array}$ & $\begin{array}{l}\text { Automatically } \\
\text { Recognizing } \\
\text { Facial } \\
\text { Expression: } \\
\text { Predicting } \\
\text { Engagement } \\
\text { and } \\
\text { Frustration }\end{array}$ \\
\hline [41] & $\begin{array}{l}\text { 2013, William } \\
\text { Hawkins, Neil } \\
\text { Heffernan, } \\
\text { Yutao Wang, } \\
\text { Ryan S.J.d. } \\
\text { Baker }\end{array}$ & $\begin{array}{l}\text { Bayesian } \\
\text { networks } \\
\text { with } \\
\text { Knowledge } \\
\text { Tracing }\end{array}$ & $\begin{array}{l}\text { Extending the } \\
\text { Assistance } \\
\text { Model: } \\
\text { Analyzing the } \\
\text { Use of } \\
\text { Assistance } \\
\text { over Time }\end{array}$ \\
\hline [42] & $\begin{array}{l}2012, \\
\text { Osmanbegovi } \\
\text { ć, Edin, and } \\
\text { Mirza Suljić }\end{array}$ & $\begin{array}{l}\text { Chi-Square } \\
\text { Test, One } \\
\text { RTest, Info } \\
\text { Gain and }\end{array}$ & $\begin{array}{l}\text { Found } \\
\text { predicting } \\
\text { model for } \\
\text { academic }\end{array}$ \\
\hline
\end{tabular}

\begin{tabular}{|c|c|c|c|}
\hline & & $\begin{array}{l}\text { Ratio Test, } \\
\text { Naive } \\
\text { Bayes, } \\
\text { DTree }\end{array}$ & $\begin{array}{l}\text { performance } \\
\text { that is user } \\
\text { friendly for } \\
\text { professors or } \\
\text { non-expert } \\
\text { users. }\end{array}$ \\
\hline [43] & $\begin{array}{l}\text { 2012, } \\
\text { Sukanya, M., } \\
\text { S. Biruntha, } \\
\text { Dr S. Karthik, } \\
\text { and T. } \\
\text { Kalaikumaran }\end{array}$ & $\begin{array}{l}\text { Bayesian } \\
\text { Classificatio } \\
\text { n Method }\end{array}$ & $\begin{array}{l}\text { Analyzed and } \\
\text { assisted the } \\
\text { low academic } \\
\text { achievers in } \\
\text { higher } \\
\text { education. }\end{array}$ \\
\hline [44] & $\begin{array}{l}2011, \\
\text { Torenbeek, } \\
\text { M., E. P. W. } \\
\text { A. Jansen, and } \\
\text { W. H. A. } \\
\text { Hofman }\end{array}$ & $\begin{array}{l}\text { Structural } \\
\text { Equations } \\
\text { Modeling, } \\
\text { Correlation } \\
\text { Matrix }\end{array}$ & $\begin{array}{l}\text { Examined two } \\
\text { variables, } \\
\text { Pedagogical } \\
\text { approach and } \\
\text { skill } \\
\text { development } \\
\text { in the first } 10 \\
\text { weeks of } \\
\text { enrollment }\end{array}$ \\
\hline [45] & $\begin{array}{l}2011, \\
\text { Yongqiang, } \\
\text { He, and } \\
\text { Zhang Shunli }\end{array}$ & $\begin{array}{l}\text { Association } \\
\text { Rules } \\
\text { Analysis }\end{array}$ & $\begin{array}{l}\text { Guidance } \\
\text { provided for } \\
\text { scientific } \\
\text { management } \\
\text { and } \\
\text { comprehensiv } \\
\text { e evaluation of } \\
\text { students. }\end{array}$ \\
\hline [46] & $\begin{array}{l}\text { 2011, Sakurai, } \\
\text { Yoshitaka, } \\
\text { Tsuruta, and } \\
\text { Rainer Knauf }\end{array}$ & $\begin{array}{l}\text { Decision } \\
\text { Tree }\end{array}$ & $\begin{array}{l}\text { Estimated } \\
\text { success } \\
\text { chances of } \\
\text { curricula by } \\
\text { implementing } \\
\text { student } \\
\text { profiling with } \\
\text { storyboard } \\
\text { system }\end{array}$ \\
\hline [47] & $\begin{array}{l}\text { 2011, Aher, } \\
\text { Sunita B., and } \\
\text { L. M. R. J. } \\
\text { Lobo }\end{array}$ & $\begin{array}{l}\text { Classificatio } \\
\mathrm{n} \text { and } \\
\text { Clustering }\end{array}$ & $\begin{array}{l}\text { Analyzed the } \\
\text { performance } \\
\text { of final year } \\
\text { students. }\end{array}$ \\
\hline [48] & $\begin{array}{l}\text { 2011, Sharma, } \\
\text { Mamta, and } \\
\text { Monali } \\
\text { Mavani }\end{array}$ & $\begin{array}{l}\text { Decision } \\
\text { Tree, Sota, } \\
\text { Naïve Bayes }\end{array}$ & $\begin{array}{l}\text { Comparison } \\
\text { of three } \\
\text { algorithms in } \\
\text { terms of } \\
\text { prediction of } \\
\text { students } \\
\text { result. }\end{array}$ \\
\hline [49] & $\begin{array}{l}\text { 2010, Ayesha, } \\
\text { Shaeela, } \\
\text { Tasleem, } \\
\text { Ahsan, Inayat }\end{array}$ & $\begin{array}{l}\text { K-Means } \\
\text { Clustering }\end{array}$ & $\begin{array}{l}\text { Analyzed } \\
\text { students' } \\
\text { learning } \\
\text { behavior to } \\
\text { check the } \\
\text { performance } \\
\text { of students } \\
\text { and predicted } \\
\text { weak students. }\end{array}$ \\
\hline [50] & $\begin{array}{l}2010, \\
\text { Kovacic, } \\
\text { Zlatko }\end{array}$ & $\begin{array}{l}\text { Classificatio } \\
\text { n Tree } \\
\text { Models }\end{array}$ & $\begin{array}{l}\text { Investigated } \\
\text { enrolment } \\
\text { attributes to } \\
\text { pre-identify } \\
\text { success of } \\
\text { students. }\end{array}$ \\
\hline [51] & $\begin{array}{l}\text { 2010, Al- } \\
\text { shargabi, }\end{array}$ & $\begin{array}{l}\text { Clustering, } \\
\text { Association }\end{array}$ & $\begin{array}{l}\text { Analyzed } \\
\text { students' }\end{array}$ \\
\hline
\end{tabular}




\begin{tabular}{|c|c|c|c|}
\hline & $\begin{array}{l}\text { Asma A., and } \\
\text { Ali N. Nusari }\end{array}$ & $\begin{array}{l}\text { Rules and } \\
\text { Decision } \\
\text { Trees }\end{array}$ & $\begin{array}{l}\text { academic } \\
\text { achievement, } \\
\text { students' drop } \\
\text { out, and } \\
\text { students' } \\
\text { financial } \\
\text { behavior. }\end{array}$ \\
\hline [52] & $\begin{array}{l}\text { 2010, Yan, } \\
\text { Zhi-min, Qing } \\
\text { Shen, and Bin } \\
\text { Shao }\end{array}$ & $\begin{array}{l}\text { Rough Set } \\
\text { Theory }\end{array}$ & $\begin{array}{l}\text { Students' } \\
\text { grades were } \\
\text { analyzed. }\end{array}$ \\
\hline [53] & $\begin{array}{l}2010, \\
\text { Ningning, } \\
\text { Gao }\end{array}$ & $\begin{array}{l}\text { Neural } \\
\text { Network, } \\
\text { Rough Set } \\
\text { Theory }\end{array}$ & $\begin{array}{l}\text { Predicted drop } \\
\text { outs from } \\
\text { course }\end{array}$ \\
\hline [54] & $\begin{array}{l}\text { 2010, Knauf, } \\
\text { Rainer, } \\
\text { Yoshitaka } \\
\text { Sakurai, } \\
\text { Setsuo } \\
\text { Tsuruta, and } \\
\text { Kouhei } \\
\text { Takada }\end{array}$ & $\begin{array}{l}\text { Decision } \\
\text { Tree }\end{array}$ & $\begin{array}{l}\text { Analyzed } \\
\text { successful } \\
\text { Storyboard (e- } \\
\text { learning } \\
\text { system) } \\
\text { success paths } \\
\text { for students. }\end{array}$ \\
\hline [55] & $\begin{array}{l}\text { 2010, Wu, X., } \\
\text { Zhang, H., \& } \\
\text { Zhang, H. }\end{array}$ & $\begin{array}{l}\text { Decision } \\
\text { Tree }\end{array}$ & $\begin{array}{l}\text { Suggested } \\
\text { comprehensiv } \\
\text { e evaluation } \\
\text { method of that } \\
\text { can } \\
\text { objectively } \\
\text { distinguish the } \\
\text { grades of } \\
\text { students. }\end{array}$ \\
\hline [56] & $\begin{array}{l}2010, \\
\text { Youping, } \\
\text { Bian } \\
\text { Xiangjuan } \\
\text { Gong } \\
\end{array}$ & $\begin{array}{l}\text { Decision } \\
\text { Tree }\end{array}$ & $\begin{array}{l}\text { Evaluated the } \\
\text { high school } \\
\text { students and } \\
\text { studying } \\
\text { effectiveness. }\end{array}$ \\
\hline [57] & $\begin{array}{l}\text { 2010, Liu, } \\
\text { Zhiwu, and } \\
\text { Xiuzhi Zhang }\end{array}$ & $\begin{array}{l}\text { Decision } \\
\text { Tree }\end{array}$ & $\begin{array}{l}\text { Built } \\
\text { forecasting } \\
\text { model for } \\
\text { students' } \\
\text { marks to } \\
\text { identify } \\
\text { negative } \\
\text { learning habits } \\
\text { or behaviors } \\
\text { of students. }\end{array}$ \\
\hline
\end{tabular}

\section{Research Objectives}

The main objective of the paper is to identify related researches in the field of EDM ranging from 2010-2017. Specifically, it sought to identify the:

1. Trends and future vision of EDM methodologies and techniques.

2. Trends and future vision of EDM tools.

3. Trends and future vision of EDM educational outcomes.

\section{RESEARCH METHODOLOGY}

This paper is a descriptive study using survey analysis of researches available from journals, and secondary data from various sources like application websites and website articles.

\section{RESULTS AND FINDINGS}

Past studies reveal the interesting field of educational data mining as a topic for research. The salient features of the features can be summarized below:

\subsection{Trends and Future Vison of EDM Methodologies and Techniques}

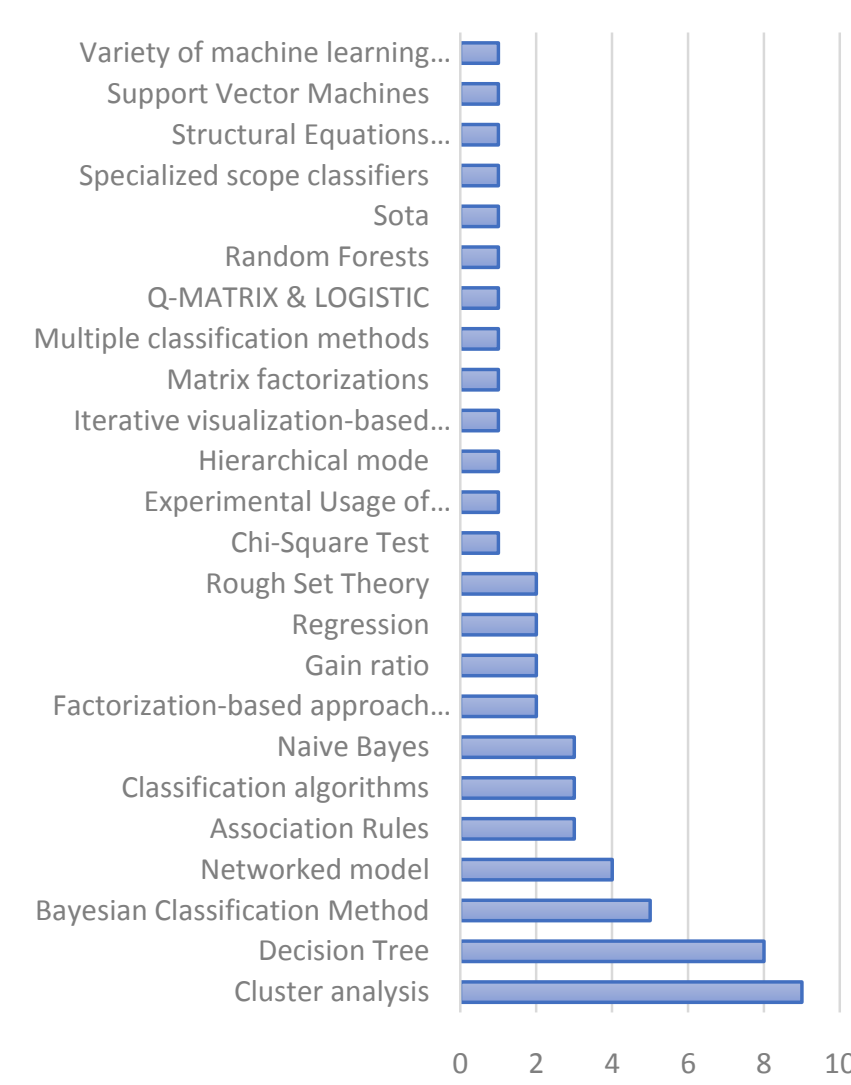

Figure 2. Trends of EDM Methodologies and Techniques During 2010-2011 as presented in figure 2, the highest research involves decision trees. Changes in data mining methods and technique are seen during the periods of 20122015. During this period, highest research involve is clustering, however, it worth noting that methods used were in a combination of more than one technique.

This trend continues to be seen from the periods of 2015 to 2017, however, this time, more complex implementation was made like further factoring and iterating the basic methods.

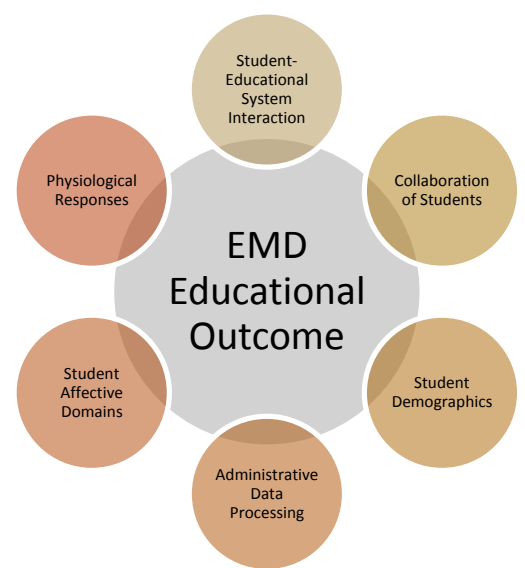

Figure 3. Journey of EDM Education Outcomes 
Now and in the future data of interest as presented in figure 3 is not restricted to interactions of individual students with an educational system alone(navigation behavior, input to quizzes and interactive exercises) but might also include data from collaborating students (chat, forums, messages), administrative data (school, district, teacher, campus), and demographic data (gender, age, school grades). Data on student affective domain (motivation, emotional states) and physiological responses (facial expression, seat posture, blinking of the eyes, perspiration, and heartbeat) are also interesting areas of research.

\subsection{Trends and Future Vision of EDM Educational Outcomes}

During 2010-2015, topic themes revolve around forecasting and analysis students' behavior, evaluation of student academic performance, estimation of success and effectiveness of program or undertakings, and comparison of predictions.

The paradigm shift of the EDM research has been seen during 2016. During this period, noteworthy researches were prediction and forecasting patterns in educational videos, analysis of problem-solving behavior in games, and effective responses of learning using data generated from face detection devices.

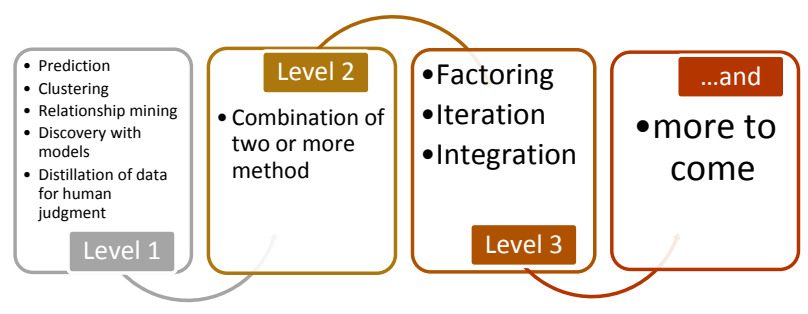

Figure 4. Journey of EDM Education Outcomes

The basic methodologies are still there as the foundation of research as shown in figure 4. However as shown earlier, researchers have combined the basic techniques to come up with meaningful research. And, it does not stop from there, as presented in the paper, researchers are applying further statistical computations like factoring, iteration, integration and the that goes along with complex education goals as presented in the earlier slides.

\subsection{Trends and Future Vision of EDM \\ Tools}

DM tools are required to validate the large set of data collected from heterogeneous environments. During 20102017 it is found that researcher mostly preferred open source tool like WEKA and then a commercial tool such as SPSS Clementine to validate their dataset.

As mentioned, these tools will not be enough to support future EDM studies and research thus the law will find its new limits. New tools need to be developed to address the growing needs in the following areas:

1. Network Computing

2. Cloud Computing

3. Stream and Distributed Data Processing

4. Internet of Things
Furthermore, user interface designs will be more complicated as PC will fade out. The text will be replaced by the new form of data like videos and images.

\section{CONCLUSION AND RECOMMENDATION}

It is always a constant challenge to educational institutions to make the learning process effective allowing full learning on the side of the students and effective delivery on the side of the teachers. Thus educational institutions make its way to innovate itself and come forward to provide the best and quality learning environment, and one of them is to evaluate and analyze existing data of students find similarity, look for differences and patterns, and predict learning performance and behavior of students.

This research is a valuable paper that contains related researches in the field of educational data mining. It presents the journey of research and practice from the year 2010 to 2017. This work focuses on research trends in data mining tools, techniques, and outcomes in an educational context and its future vision.

As an extension of this work in the future is to build an EDM framework taking into consideration the specific application of tools and techniques that will use by any college to strengthen the utilization of existing resources.

\section{ACKNOWLEDGEMENT}

The authors wish to thank Salalah College of Technology for the continuous support on this undertaking.

\section{REFERENCES}

[1]"educationaldatamining.org", Educationaldatamining.org, 2017. [Online]. Available: http://educationaldatamining.org/. [Accessed: 13- Dec2017].

[2]"Mining educational data to analyze learning and teaching methods, the case of medicine | PersyvalLab", Persyval-lab.org, 2017. [Online]. Available: https://persyval-lab.org/en/exploratoryproject/Med_LT_med. [Accessed: 13- Dec- 2017].

[3]A. Francisco, "Realizing the Opportunity for Big Data in Education - Digital Promise", Digital Promise, 2017. [Online]. Available: http://digitalpromise.org/2014/04/17/realizing-theopportunity-for-big-data-in-education/. [Accessed: 13Dec- 2017].

[4]"Data Mining Classification \& Prediction", www.tutorialspoint.com, 2017. [Online]. Available:

https://www.tutorialspoint.com/data_mining/dm_classifi cation_prediction.htm. [Accessed: 14- Dec- 2017].

[5]"What is Clustering in Data Mining?", Big Data Made Simple - One source. Many perspectives., 2017. [Online]. Available: http://bigdatamadesimple.com/what-is-clustering-in-data-mining/. [Accessed: 14- Dec- 2017].

[6]C. Rygielski, J. Wang and D. Yen, "Data mining techniques for customer relationship management", 2017. .

[7]"Educational data mining - EduTech Wiki", Edutechwiki.unige.ch, 2017. [Online]. Available: 
http://edutechwiki.unige.ch/en/Educational_data_minin g. [Accessed: 14- Dec- 2017].

[8]D. Thanamani, "An Overview of Knowledge Discovery Databaseand Data mining Techniques", Rroij.com, 2017. [Online]. Available: http://www.rroij.com/openaccess/an-overview-of-knowledge-discoverydatabaseand-data-mining-techniques.php?aid $=48833$. [Accessed: 14- Dec- 2017].

[9]"MS SQL Server Tutorial",www.tutorialspoint.com, $2017 . \quad$ [Online]. Available: https://www.tutorialspoint.com/ms_sql_server/. [Accessed: 14- Dec- 2017].

[10]"Oracle Data Mining", Oracle.com, 2017. [Online]. Available:

http://www.oracle.com/technetwork/database/options/ad vanced-analytics/odm/overview/index.html. [Accessed: 14- Dec- 2017].

[11]D. science and P. analytics, "IBM SPSS Software | IBM Analytics", Ibm.com, 2017. [Online]. Available: https://www.ibm.com/analytics/data-science/predictiveanalytics/spss-statistical-software. [Accessed: 14- Dec2017].

[12]"Weka 3 - Data Mining with Open Source Machine Learning Software in Java", Cs.waikato.ac.nz, 2017. [Online]. Available: https://www.cs.waikato.ac.nz/ml/weka/. [Accessed: 14Dec- 2017].

[13]"Orange - Data Mining Fruitful \& Fun", Orange.biolab.si, 2017. [Online]. Available: https://orange.biolab.si/. [Accessed: 14- Dec- 2017].

[14]D. Weiss, "Carrot2 - Open Source Search Results Clustering Engine", Project.carrot2.org, 2017. [Online]. Available: https://project.carrot2.org/. [Accessed: 14Dec- 2017].

[15]G. Kavitha and L. Raj, "Educational Data Mining and Learning Analytics Educational Assistance for Teaching and Learning", International Journal of Computer \& organization Trends, vol. 41, no. 1, pp. 21-25, 2017.

[16]J. R R ihák and R. Pelánek, "Measuring Similarity of Educational Items Using Data on Learners' Performance", 10th International Conference on Educational Data Mining, China, 2017.

[17]J. R ihák and R. Pelánek, "Measuring Similarity of Educational Items Using Data on Learners' Performance", 10th International Conference on Educational Data Mining, China, 2017.

[18]Z. Ren, X. Ning and H. Rangwala, "Grade Prediction with Temporal Course-wise Influence", in 10th International Conference on Educational Data Mining, China, 2017.

[19]A. Stewart, N. Bosch and S. D’Mello, "Generalizability of Face-Based Mind Wandering Detection Across Task Contexts", 10th International Conference on Educational Data Mining, China, 2017.

[20]A. Olney, D. Bakhtiari, D. Greenberg and A. Graesser, "Assessing Computer Literacy of Adults with Low Literacy Skills", in 10th International Conference on Educational Data Mining, China, 2017.
[21]R. Balyan, K. McCarthy and D. McNamara, "Combining Machine Learning and Natural Language Processing to Assess Literary Text Comprehension", in 10th International Conference on Educational Data Mining, China, 2017.

[22]A. Katare and S. Dubey, "A Study of various Techniques for Predicting student Performance under Educational Data Mining", International Journal of Electrical, Electronics and Computer Engineering, 2016.

[23]A. Abu, "Educational Data Mining \& Students' Performance Prediction", International Journal of Advanced Computer Science and Applications, vol. 7, no. $5,2016$.

[24]A. Vail, J. Wiggins, J. Grafsgaard, K. Boyer, E. Wiebe and J. Lester, "The Affective Impact of Tutor Questions: Predicting Frustration and Engagement", 9th International Conference on Educational Data Mining, 2016.

[25]M. Stapel, Z. Zheng and N. Pinkwart, "An Ensemble Method to Predict Student Performance in an Online Math Learning Environment", in 9th International Conference on Educational Data Mining, 2016.

[26]M. Saarela and T. K ärkkäinen, "Analysing Student Performance using Sparse Data of Core Bachelor Courses", Journal of Educational Data Mining, vol. 7, no. $1,2016$.

[27]A. Sharma, A. Biswas, A. Gandhi, S. Patil and O. Deshmukh, "LIVELINET: A Multimodal Deep Recurrent Neural Network to Predict Liveliness in Educational Videos", in 9th International Conference on Educational Data Mining, 2016.

[28]K. Niu, Z. Niu, X. Zhao, C. Wang, K. Kang and M. Ye, "A Coupled User Clustering Algorithm for Web-based Learning Systems", in 9th International Conference on Educational Data Mining, 2016.

[29]E. Bumbacher, S. Salehi, M. Wierzchula and P. Blikstein, "Learning Environments and Inquiry Behaviors in Science Inquiry Learning: How their Interplay Affects the Development of Conceptual Understanding in Physics", in 8th International Conference on Educational Data Mining, Spain, 2015.

[30]J. Nižnan, R. Pelánek and J. R` ihák, "Student Models for Prior Knowledge Estimation", in 8th International Conference on Educational Data Mining, Spain, 2015.

[31]Y. Chen, P. Wuillemin and J. Labat, "Discovering Prerequisite Structure of Skills through Probabilistic Association Rules Mining", in 8th International Conference on Educational Data Mining, Spain, 2015.

[32]A. Ezen-Can and K. Boyer, "Choosing to Interact: Exploring the Relationship Between Learner Personality, Attitudes, and Tutorial Dialogue Participation", in 8th International Conference on Educational Data Mining, Spain, 2015.

[33]M. Saarela and T. Kärkkäinen, "Do Country Stereotypes Exist in PISA? A Clustering Approach for Large, Sparse, and Weighted Data.", in 8th International Conference on Educational Data Mining, Spain, 2015.

[34] Peña-Ayala, Alejandro. "Educational data mining: A survey and a data mining-based analysis of recent 
works." Expert systems with applications 41.4 (2014): $1432-1462$

[35] Saranya, S., R. Ayyappan, and N. Kumar. "Student Progress Analysis and Educational Institutional Growth Prognosis Using Data Mining." International Journal Of Engineering Sciences \& Research Technology, 2014

[36] Archer, Elizabeth, Yuraisha Bianca Chetty, and Paul Prinsloo. "Benchmarking the habits and behaviors of successful students: A case study of academic-business collaboration." The International Review of Research in Open and Distance Learning 15.1 (2014).

[37] Hicheur Cairns, Awatef, et al. "Towards CustomDesigned Professional Training Contents and Curriculums through Educational Process Mining." IMMM 2014, The Fourth International Conference on Advances in Information Mining and Management. 2014.

[38] Arora, Rakesh Kumar, and Dharmendra Badal. "Mining Association Rules to Improve Academic Performance." (2014).

[39]S. Fancsali, T. Nixon and S. Ritter, "Optimal and WorstCase Performance of Mastery Learning Assessment with Bayesian Knowledge Tracing", in International Conference on Educational Data Mining, USA, 2013.

[40]J. Grafsgaard, J. Wiggins, K. Boyer, E. Wiebe and J. Lester, "Automatically Recognizing Facial Expression: Predicting Engagement and Frustration", in International Conference on Educational Data Mining, USA, 2013

[41]W. Hawkins, N. Heffernan, Y. Wang and R. Baker, "Extending the Assistance Model: Analyzing the Use of Assistance over Time", in International Conference on Educational Data Mining, USA, 2013

[42] Osmanbegović, Edin, and Mirza Suljić. "Data mining approach for predicting student performance." Economic Review 10.1 (2012).

[43] Sukanya, M., S. Biruntha, Dr S. Karthik, and T. Kalaikumaran. "Data mining: Performance improvement in education sector using classification and clustering algorithm." In International conference on computing and control engineering,(ICCCE 2012), vol. 12. 2012.

[44] Torenbeek, M., E. P. W. A. Jansen, and W. H. A. Hofman. "Predicting first-year achievement by pedagogy and skill development in the first weeks at university." Teaching in Higher Education 16.6 (2011): 655-668.

[45] Yongqiang, He, and Zhang Shunli. "Application of Data Mining on Students' Quality Evaluation." Intelligent Systems and Applications (ISA), 2011 3rd International Workshop on. IEEE, 2011.

[46] Sakurai, Yoshitaka, Setsuo Tsuruta, and Rainer Knauf. "Success Chances Estimation of University Curricula Based on Educational History, Self-Estimated Intellectual Traits and Vocational Ambitions." Advanced Learning Technologies (ICALT), 2011 11th IEEE International Conference on. IEEE, 2011.

[47] Aher, Sunita B., and L. M. R. J. Lobo. "Data mining in educational system using Weka." IJCA Proceedings on
International Conference on Emerging Technology Trends (ICETT). Vol. 3. 2011.

[48] Sharma, Mamta, and Monali Mavani. "Accuracy Comparison of Predictive Algorithms of Data Mining: Application in Education Sector." Advances in Computing, Communication and Control. Springer Berlin Heidelberg, 2011. 189-194.

[49] Ayesha, Shaeela, Tasleem Mustafa, Ahsan Raza Sattar, and M. Inayat Khan. "Data mining model for higher education system." Europen Journal of Scientific Research 43, no. 1 (2010): 24-29.

[50] Kovacic, Zlatko. "Early prediction of student success: Mining students' enrolment data." (2010).

[51] Al-shargabi, Asma A., and Ali N. Nusari. "Discovering vital patterns from UST students data by applying data mining techniques." Computer and Automation Engineering (ICCAE), 2010 The 2nd International Conference on. Vol. 2. IEEE, 2010.

[52] Yan, Zhi-min, Qing Shen, and Bin Shao. "The analysis of student's grade based on Rough Sets." Ubi-media Computing (U-Media), 2010 3rd IEEE International Conference on. IEEE, 2010.

[53] Ningning, Gao. "Proposing Data Warehouse and Data Mining in Teaching Management Research." Information Technology and Applications (IFITA), 2010 International Forum on. Vol. 1. IEEE, 2010.

[54] Knauf, Rainer, Yoshitaka Sakurai, Setsuo Tsuruta, and Kouhei Takada. "Empirical evaluation of a data mining method for success chance estimation of university curricula." In Systems Man and Cybernetics (SMC), 2010 IEEE International Conference on, pp. 1127-1133. IEEE, 2010.

[55] Wu, X., Zhang, H., \& Zhang, H. (2010, October). Study of comprehensive evaluation method of undergraduates based on data mining. In Intelligent Computing and Integrated Systems (ICISS), 2010 International Conference on(pp. 541-543). IEEE.

[56] Youping, Bian Xiangjuan Gong. "The application of data mining technology in analysis of college student's performance." Information Science (2010).

[57] Liu, Zhiwu, and Xiuzhi Zhang. "Prediction and Analysis for Students' Marks Based on Decision Tree Algorithm." Intelligent Networks and Intelligent Systems (ICINIS), 2010 3rd International Conference on. IEEE, 2010.

\section{AUTHOR PROFILE}

Mr. Bernard Ugalde is a graduate of Bachelor of Science in Computer Science, and master degree holders in Information Technology and MA in College Teaching.

Dr. R. Venkateswaran received his professional degree MCA from Bharathiar University, Tamilnadu, India, He received his M.Phil in computer science from Bharathidasan University, Tamilnadu, India, and Ph.D in Computer Science with Informaiton Security Specialization from Karpagam Academy of Higher Education, Karpagam University, Tamilnadu, India in the field of Cryptography and Network Security. Presently he is the faculty of Networking and Information Security, IT Department, Salalah College of 
Technology, Salalah, OMAN from the Year 2012. He has 15 years of teaching experience and 3 years of research experience. He has participated in many national level conferences and workshops, published papers in several international conferences and proceedings. He has Published 8 papers in international refereed journals. He is a member of
CSI, IAENG, IACSIT, CSTA and many online forums. He has completed Oracle 9i at Oracle University and $\mathrm{He}$ is Cisco Certified CCNA Professional. His research interests are in cryptography and network security, information security, software engineering and database management systems. 